Polymer Journal, Vol. 38, No. 9, pp. 976-982 (2006)

(C) 2006 The Society of Polymer Science, Japan

\title{
Polyacetylene Intermediate Bearing Reactive Benzylidene Malonate: Helix Induction, Inversion, and Recovery by Tandem Michael and Amidation Reactions with Chiral Nucleophiles and Water
}

\author{
Giseop KwaK, ${ }^{1, \dagger}$ Shin-Ichi Hososhima, ${ }^{2}$ and Michiya FuJIKI ${ }^{2, \dagger}$ \\ ${ }^{1}$ Department of Polymer Science, Kyungpook National University, \\ 1370 Sankyuk-dong, Buk-ku, Daegu 702-701, Korea \\ ${ }^{2}$ Graduate School of Materials Science, Nara Institute of Science and Technology, \\ 8916-5 Takayama, Ikoma 630-0101, Japan
}

(Received May 9, 2006; Accepted June 29, 2006; Published August 11, 2006)

\begin{abstract}
A new polyacetylene bearing benzylidene malonate group, poly[4-\{(diethyl- $\beta$-malonyl)vinyl $\}$ phenylacetylene], 1, was designed as a prochiral reacting polyacetylene intermediate to provide helix induction and inversion abilities with chiral nucleophiles. Polymer 1 underwent both Michael reaction and amidation in the presence of $(R)$-2-amino-1-propanol $(R)$-3 (and its $(S)$-form) to afford optically active polymers with extremely complicated and optically active sites in the side chains. UV-vis, circular dichroism, and NMR studies of 1 revealed the rapid coilto-helix and subsequent slow helix-to-helix transitions of the resulting optically active polymers. Although the inverted helical conformation was fairly stabilized by intramolecular hydrogen bonds between the complicated and optically active side chains, the inverted helical sense was readily recovered to the initial screw sense by adding a very little amount of water. Polymer 1 is applicable to new chiroptical switching induced by various chiral nucleophiles and sensory systems to detect a trace amount of water. Polymer 1 may thus be a very useful prochiral precursor polymer for preparing various functional and biomimetic helical and higher ordered polymers in future.

[doi:10.1295/polymj.PJ2006027]

KEY WORDS Benzylidene Malonate / Polyacetylene / Helix / Michael Reaction / Amidation /

Circular Dichroism / Optically Active /
\end{abstract}

Michael reaction is one of the most fundamental reactions in organic chemistry. Among a variety of unsaturated electrophiles, benzylidene malonate has been described as an excellent Michael acceptor in the conjugate addition. ${ }^{1}$ Especially, in most catalytic enantioselective syntheses with use of $C_{2}$-symmetric $\mathrm{Cu}$ (II) complexes, the malonate derivative has been used for the preparation of various chiral compounds, as a starting material..$^{2-7}$ Owing to this reaction, organic chemists have developed a number of biologically and optically active compounds. Moreover, the malonate ester may further undergo ester amidation in the presence of nucleophiles such as primary amines, although it is kinetically slow compared to Michael reaction. In spite of the synthetic versatileness and usefulness, little attention has been paid to the application of both reactions in polymer chemistry.

Recently, a great number of synthetic, helical polymers which mimic biological systems have been developed to help us understand the molecular chirality recognition mechanisms. ${ }^{8-19}$ Incorporation of such functional groups as amine, hydroxylamine, amide, amino acid, and saccharide into the synthetic polymers is of particular importance from the view point of exploiting new biological applications. Except for several helical polymers induced by interaction with chiral molecules, ${ }^{8-19}$ most synthetic methodologies of such polymers have been step-by-step reactions from starting materials containing such biologically and optically active functional groups to the target polymers via the monomers. Thus, it could be a very challenging issue to design and develop a new polymer intermediate bearing reacting side groups available for the facile preparation of such polymers targeted for biological applications.

On the basis of this idea, we designed and synthesized a new polyacetylene derivative containing benzylidene malonate in the side chain. The polymer underwent Michael reaction followed by ester amidation with $(R)$ - and (S)-2-amino-1-propanols, leading to its helix induction and helix-sense inversion. The inverted helix-sense was, however, recovered to the initial helix-sense by a trace of amount of water.

\section{EXPERIMENTAL}

\author{
Synthesis of Monomer \\ 4-[(Diethyl- $\beta$-malonyl)vinyl]phenylacetylene (2): A
}

†To whom correspondence should be addressed (E-mail: gkwak@knu.ac.kr (GK); fujikim@ms.naist.jp (MF)). 


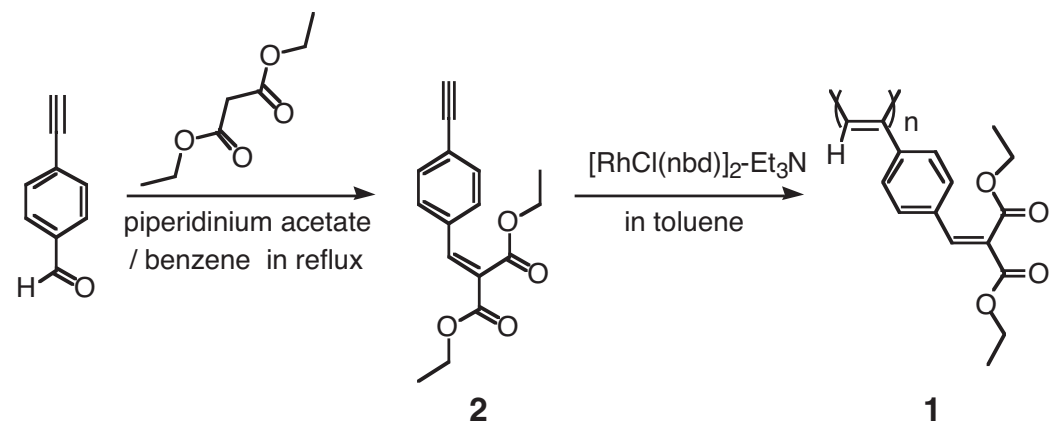

Scheme 1. Synthesis of poly[4-\{(diethyl- $\beta$-malonyl)vinyl $\}$ phenylacetylene], 1 , and the corresponding monomer, 2.

$50 \mathrm{~mL}$ round-bottomed flask was equipped with a dropping funnel, a three-way stopcock, and a magnetic stirring bar and flushed with dry nitrogen gas. 4-Ethynylbenzaldehyde $(0.71 \mathrm{~g}, 5.45 \mathrm{mmol})$, diethyl malonate $(0.87 \mathrm{~g}, 5.45 \mathrm{mmol})$, dry benzene $(5.0 \mathrm{~mL})$, and a catalytic amount of piperidinium acetate $(0.1 \mathrm{~g})$ were placed in the flask and refluxed overnight. The solvent and volatiles were evaporated under reduced pressure, and the crude product was purified by flash column chromatography (Merck, silica gel 60; eluent, hexane/ethyl acetate $=20 / 1$ ) to give the desired product (yield $1.10 \mathrm{~g}, 75 \%$ ) as an yellow solid. IR (KBr): 3280, 2983, 2938, 2902, 1727, 1630, 1373, 1259, 1207, 1066, 1020, $829 \mathrm{~cm}^{-1} \cdot{ }^{1} \mathrm{H}$ NMR $\left(\mathrm{CDCl}_{3}\right.$,

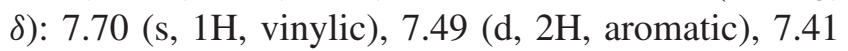
(d, $2 \mathrm{H}$, aromatic), 4.33 (m, $4 \mathrm{H}$, ethylene), $1.31(\mathrm{~m}$, $6 \mathrm{H}$, methyl) ppm. ${ }^{13} \mathrm{C} \mathrm{NMR}\left(\mathrm{CDCl}_{3}, \delta\right): 166.4,141.0$, 133.2, 132.4, 129.3, 127.1, 124.2, 82.9, 79.5, 61.8, 14.1 ppm. Anal. Calcd for $\mathrm{C}_{16} \mathrm{H}_{16} \mathrm{O}_{4}: \mathrm{C}, 70.57 ; \mathrm{H}$, 5.92. Found: C, 70.38; H, 5.79.

\section{Synthesis of Polymer}

Poly[4-\{(diethyl- $\beta$-malonyl)vinyl $\}$ phenylacetylene] (1): To a solution of $2(0.1 \mathrm{~g}, 0.37 \mathrm{mmol})$ in dry THF $(4.0 \mathrm{~mL})$ at $25^{\circ} \mathrm{C}$ under nitrogen, a solution of $[\mathrm{RhCl}$ $(\mathrm{nbd})]_{2}(0.7 \mathrm{mg}, 1.52 \mu \mathrm{mol})$ and $\mathrm{Et}_{3} \mathrm{~N}(5.0 \mu \mathrm{L})$ in dry THF $(1.0 \mathrm{~mL})$ was added. The polymerization was kept at $25^{\circ} \mathrm{C}$ for $1 \mathrm{~h}$, and then poured into a large excess of dry methanol to precipitate the polymer as an orange solid. The polymer was filtered with a sintered glass filter (G3) and dried in nitrogen atmosphere and in vacuum for a few minutes. Yield: 95\%. IR (film): 2983, 2938, 2902, 1727, 1630, 1373, $1259,1207,1066,1020 \mathrm{~cm}^{-1} .{ }^{1} \mathrm{H}$ NMR (THF- $d_{8}, \delta$ ): $7.80(1 \mathrm{H}$, vinylic), $7.48(2 \mathrm{H}$, aromatic $), 6.98(2 \mathrm{H}$, aromatic), $6.13(1 \mathrm{H}$, alkenylenic), 4.63 (4H, ethylene), 1.74-1.48 (6H, methyl) ppm. ${ }^{13} \mathrm{C}$ NMR (THF- $d_{8}$, $\delta): 166.9,145.3,141.1,139.8,132.9,130.7,128.8$, 127.0, 61.9, $14.6 \mathrm{ppm}$. Anal. Calcd for $\mathrm{C}_{16} \mathrm{H}_{16} \mathrm{O}_{4}$ in repeat unit: C, 70.57; H, 5.92. Found: C, 70.82; H, 5.80 .

\section{Measurements}

${ }^{1} \mathrm{H}(400 \mathrm{MHz})$ NMR spectra were measured in $\mathrm{CDCl}_{3}$ and THF- $d_{8}$ solution at $25{ }^{\circ} \mathrm{C}$ on a JEOL EX-400 spectrometer. The weight-average molecular weight $\left(M_{\mathrm{w}}\right)$ and number-average molecular weight $\left(M_{\mathrm{n}}\right)$ of the polymers were evaluated using gel permeation chromatography (Shimadzu A10 instruments, Polymer Laboratories, PLgel Mixed-B $(300 \mathrm{~mm}$ in length) as a column, and HPLC-grade tetrahydrofuran as eluent at $40^{\circ} \mathrm{C}$ ), based on a calibration with polystyrene standards. IR, UV-vis, and circular dichroism (CD) spectra were measured on Horiba FT-730 FT-IR spectrometers, JASCO UV-550 spectrophotometers, and JASCO J-820 spectropolarimeter, respectively. Intrinsic viscosity-molecular weight relationship was performed using an in-line configuration of a viscometer (Viscotek T60A) and SEC (Shimadzu; column: Polymer Laboratories, PLgel Mixed-B $300 \mathrm{~mm}$ in length; eluent: HPLC-grade THF) instrument at $30^{\circ} \mathrm{C}$. The corresponding intrinsic viscosity as a function of molecular weight was obtained from the viscometer.

\section{RESULTS AND DISCUSSION}

Scheme 1 outlines the synthesis of poly[4-\{(diethyl- $\beta$-malonyl)vinyl $\}$ phenylacetylene], 1 , and the corresponding monomer, 2. Functionalization of diethyl malonate via Knoevenagel condensation was carried out with 4-ethynylbenzaldehyde in the presence of piperidinium acetate as a catalyst. Purification by evaporation of volatiles under reduced pressure, subsequent column chromatography afforded the monomer 2 as a pale yellow solid in a relatively high yield of $75 \%$. Polymerization of 2 by $[\mathrm{RhCl}(\mathrm{nbd})]_{2}-\mathrm{Et}_{3} \mathrm{~N}$ in THF under nitrogen atmosphere gave 1 with an extremely high molecular weight $\left(M_{\mathrm{w}}=5.3 \times 10^{5}\right.$, $M_{\mathrm{w}} / M_{\mathrm{n}}=2.9$ ), as an orange solid in a high yield of $95 \%$.

The ${ }^{1} \mathrm{H}$ NMR and IR spectra confirmed that 1 is a perfectly stereoregular polymer with an almost $100 \%$ cis-transoidal structure and no detectable structural defects. As shown in Figure 1, the ${ }^{1} \mathrm{H}$ NMR and IR 
a)

one of the expected structures
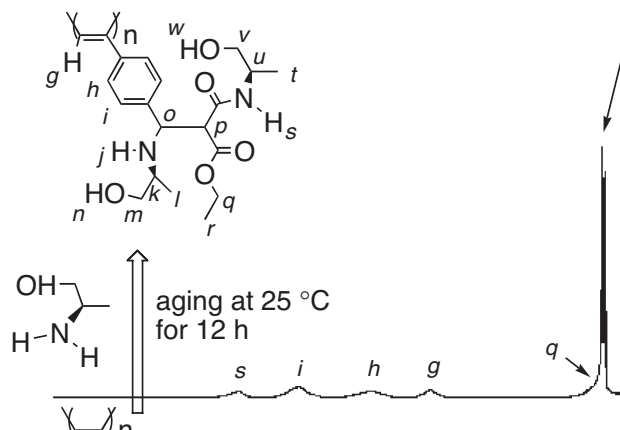

unreacted (R)-2-amino-1propanol
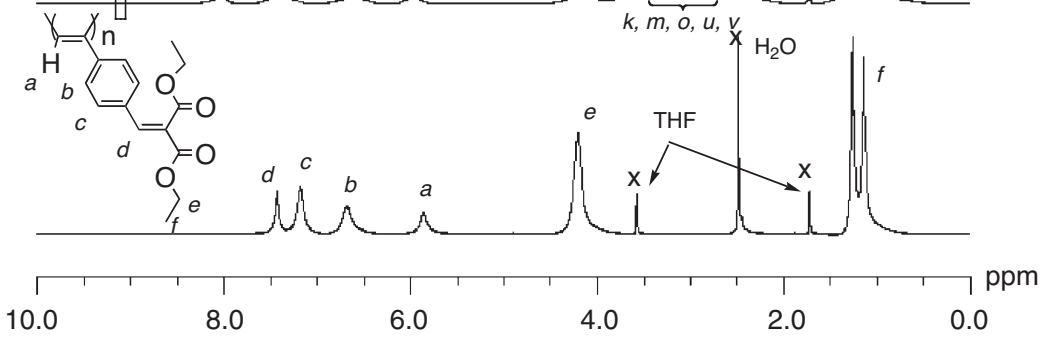

b)

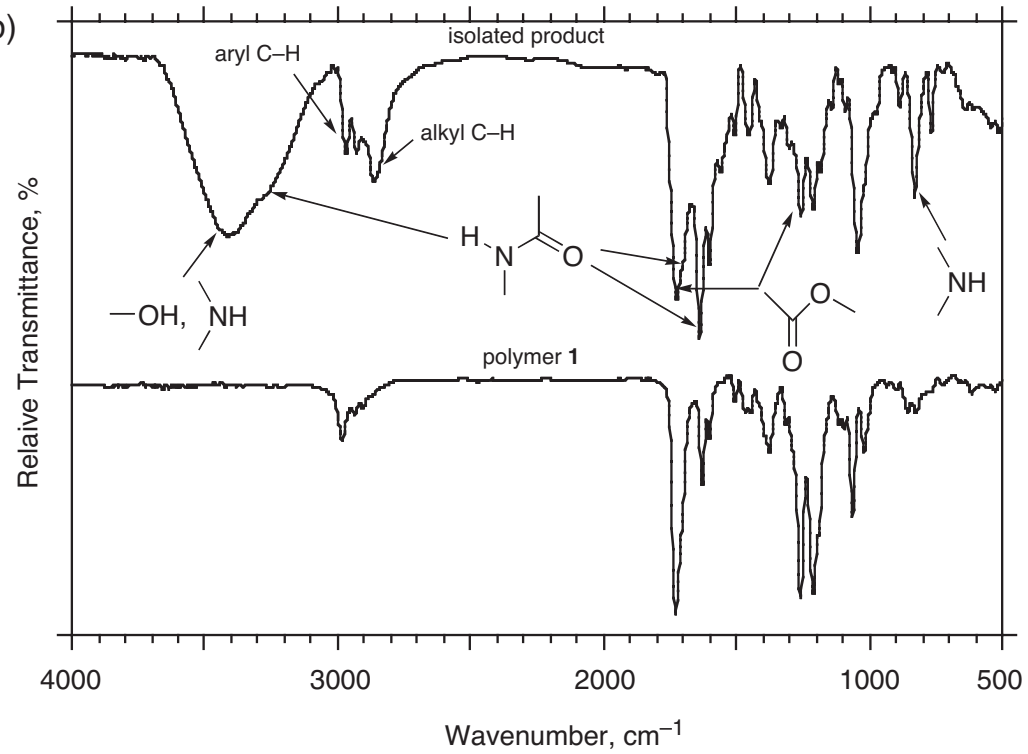

Figure 1. a) ${ }^{1} \mathrm{H}$ NMR spectra of 1 before and after treatment with 3.1 equiv of $(R)$-2-amino-1-propanol and b) IR spectra of 1 and the isolated product from the mixture $\left({ }^{1} \mathrm{H}\right.$ NMR: $[\mathrm{C}]_{0}=7.14 \times 10^{-2} \mathrm{M}$, at $25^{\circ} \mathrm{C}$ in THF- $d_{8}$; IR: cast film on $\left.\mathrm{KBr}\right)$.

spectra exhibit the well-defined structure of the polymer: the IR spectrum exhibited no absorption at $3280 \mathrm{~cm}^{-1}$ due to ethynyl group, while an intense peak at $1727 \mathrm{~cm}^{-1}$ due to carbonyl group was seen, indicating that the malonate moiety remained intact after polymerization. The ${ }^{1} \mathrm{H}$ NMR spectrum clearly showed a vinylic proton peak at $7.43 \mathrm{ppm}(d)$, ethyl proton peaks at $4.21(e), 1.21(f)$ ppm, and a characteristic cis-alkenylenic proton peak at $5.86 \mathrm{ppm}(a)$. The peak ratio of the aromatic $(b, c)$, alkenylenic $(a)$, vinylic $(d)$, and ethyl $(e, f)$ group resonances was about 3.9:1.0:1.0:10.5, which agreed well with the theoretical ratio $(4: 1: 1: 10)$. From the peak ratio of alkenylenic and aromatic protons, the cis content of the present polymer was calculated to be $>99 \%$.

Figure 1a shows the ${ }^{1} \mathrm{H}$ NMR spectra of 1 before and after treatment with 3.1 equiv of $(R)$-2-amino-1propanol, $(R)-3$. After 1 reacted with $(R)-3$ at $25^{\circ} \mathrm{C}$ for $12 \mathrm{~h}$, the vinylic proton $(d)$ peak at $7.43 \mathrm{ppm}$ completely disappeared, whereas a new peak at $3.55 \mathrm{ppm}$ due to the acidic methyn proton $(p)$ appeared, indicating that 1 completely underwent Michael reaction. Moreover, a new broad peak around $8.03 \mathrm{ppm}$ due to amide NH proton $(s)$ appeared, whereas the ethyl protons $(e, f)$ within the malonate moiety shifted toward high field (peaks $q$ and $r$ ), and significantly sharp signals at 4.20, 3.31, and $1.23 \mathrm{ppm}$ due to the ethanol protons appeared. This indicated that 1 further under- 

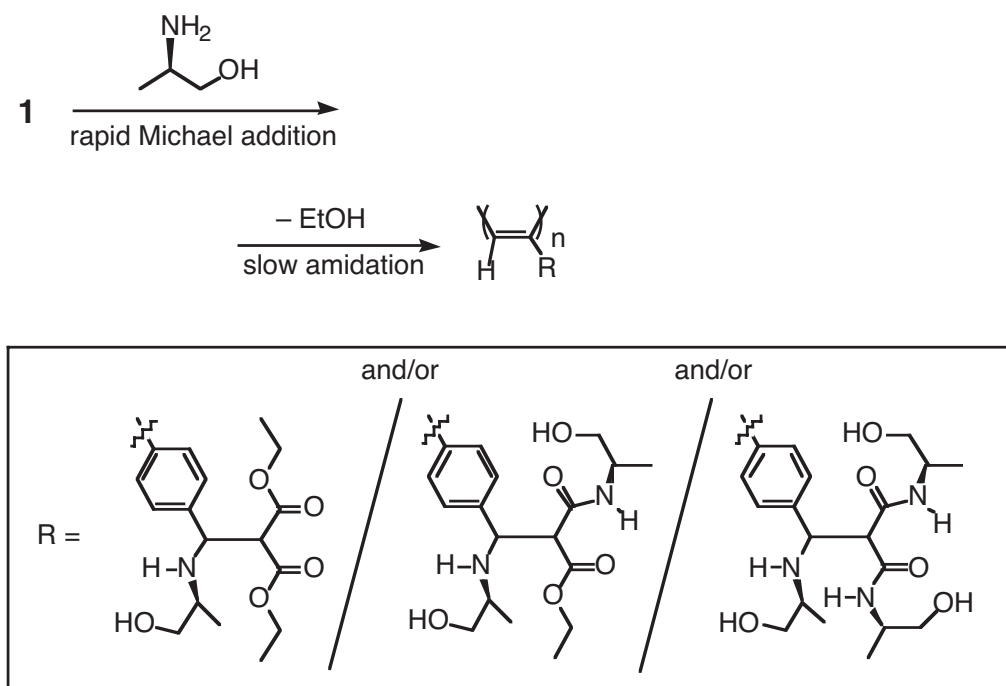

Scheme 2. Reaction of 1 with (R)-2-amino-1-propanol and proposed structures.

went ester amidation accompanied by elimination of ethanol. Also, the peaks due to aromatic $(h, i)$ and alkenylenic $(g)$ protons were remarkably broad relative to the corresponding peaks $(b, c, a)$, indicating the reduced mobility of the polymer chain.

If ester amidation thoroughly occurred, the peaks due to ethyl protons $(e, f)$ should completely disappear, and the peak ratio of amide $(s)$ and alkenylenic $(g)$ protons should be $2: 1$. However, the ethyl protons were still shown as peaks $q$ and $r$, and the peak ratio of amide and alkenylenic protons was kept to be $1: 1$ even after addition of more than 3.1 equiv of $(R)-3$. Therefore, both the reactions and proposed structures of the resulting polymer should be described as shown in Scheme 2. At the present time, the reason why ester amidation stopped at 50\% conversion is unclear. Unfortunately, we could not determine NMR spectrum of the isolated product obtained by precipitation from methanol because it was scarcely soluble in any organic solvents such as THF, chloroform, and DMSO. The insolubility is presumably due to the increased intermolecular hydrogen bonding in the solid state as well as the increased hydrophilicity.

Figure 1b compares the IR spectra of the isolated product and the original 1 . New peaks due to amide $\left(3280,1700,1637 \mathrm{~cm}^{-1}\right)$, secondary amine $(830$ $\left.\mathrm{cm}^{-1}\right)$, and hydroxy $\left(3420 \mathrm{~cm}^{-1}\right)$ groups appeared in the isolated product, while the peak due to the ester $\left(1257 \mathrm{~cm}^{-1}\right)$ group significantly decreased. Furthermore, significant increases of the peaks (2990-2790 $\mathrm{cm}^{-1}$ ) due to alkyl C-H stretching vibration were observed. These data led to the idea that 1 underwent both Michael reaction and amidation in the presence of $(R)-3$ to newly form extremely complicated and optically active sites in the side chain.

Figure 2 shows the changes in UV-vis and CD

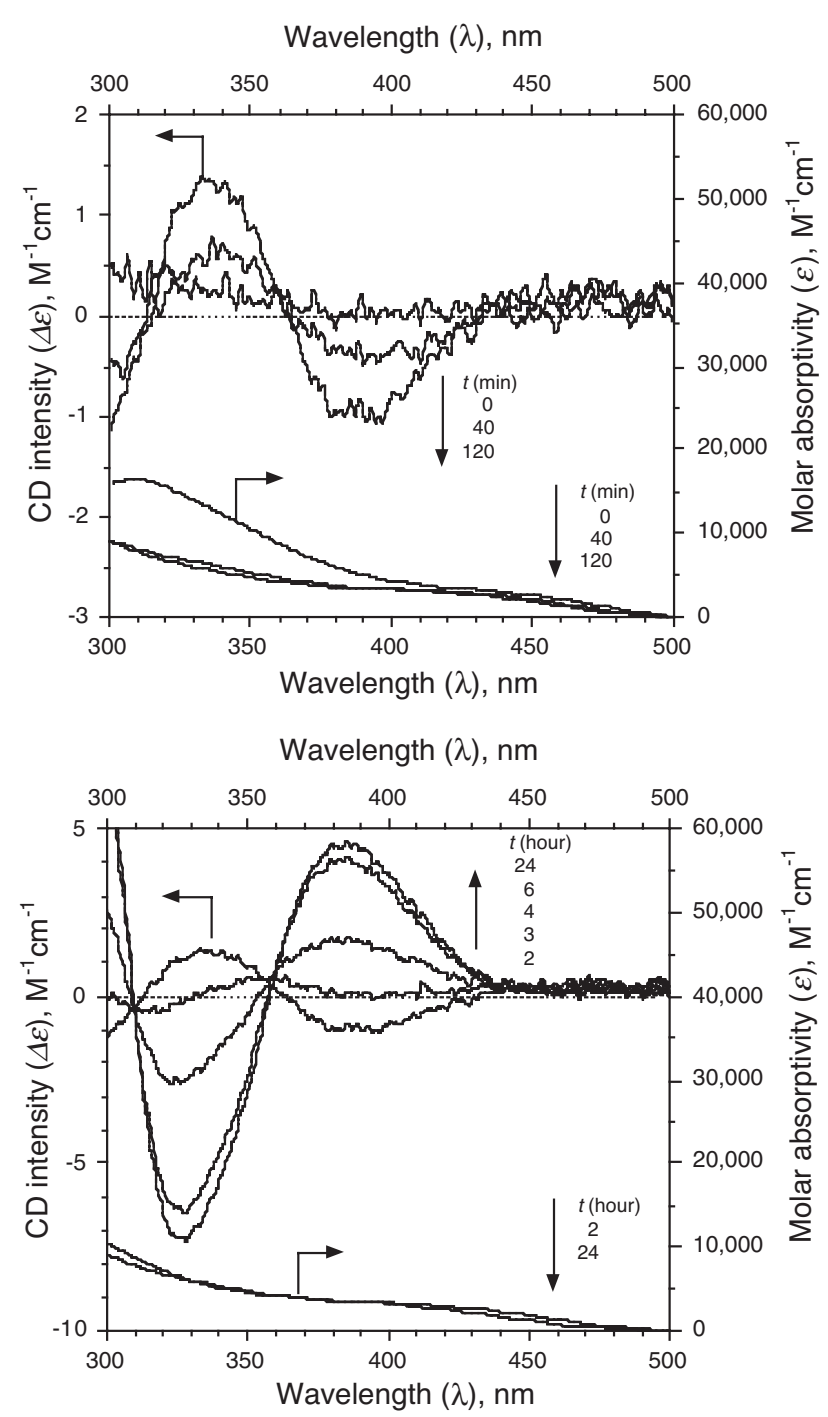

Figure 2. Changes in UV-vis and CD spectra of 1 with aging time in the presence of excess $(R)$-2-amino-1-propanol $\left([\mathrm{C}]_{0}=\right.$ $6.40 \times 10^{-4} \mathrm{M},[(R)-2$-amino-1-propanol $] /[1]=300$, at $25^{\circ} \mathrm{C}$ in THF). 


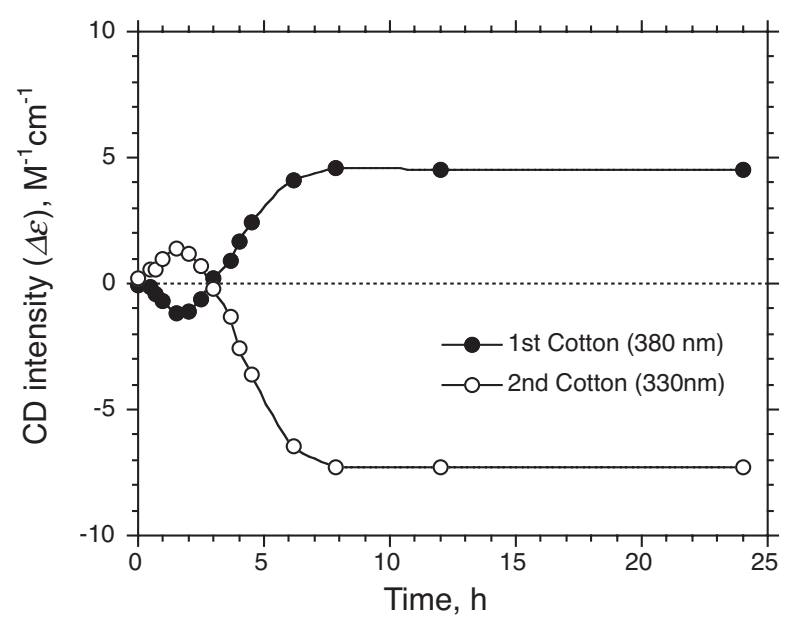

Figure 3. Time dependency of Cotton effects of 1 treated with (R)-2-amino-1-propanol.

spectra of 1 with aging time at $25^{\circ} \mathrm{C}$ in THF in the presence of $(R)-3$. After addition of $(R)-3$ to the THF solution of 1 , an intense UV absorption band at $308 \mathrm{~nm}$ due to the conjugated benzylidene malonate immediately disappeared. Subsequently, a broad UVvis absorption band in the range of $300-500 \mathrm{~nm}$ due to the $\pi-\pi^{*}$ transition of the backbone gradually shifted to shorter wavelengths with aging time. We thus assumed that the chiral nucleophile $(R)-3$ rapidly attacks the electrophilic olefin within benzylidene malonate moiety, and slowly reacts with the ester groups, leading to a marked change in secondary structure of the polymer chain. The inversion in the CD spectra supported this idea: After the addition of $(R)-3$, a splittype CD signal which has weak positive Cotton band at $325 \mathrm{~nm}$ and weak negative Cotton band at $380 \mathrm{~nm}$ immediately appeared and gradually inverted in the backbone absorption region during the initial period of about $2 \mathrm{~h}$, and very slowly and oppositely increased to reach maximum values approximately $12 \mathrm{~h}$ later. As shown in Figure 3, the initial weak positive- $325 \mathrm{~nm}$ and negative- $380 \mathrm{~nm}$ Cotton bands were eventually inverted to the corresponding intense negative- $325 \mathrm{~nm}$ and positive- $380 \mathrm{~nm}$ Cotton bands, respectively, with a prolonged reaction time with $(R)-3$. These data suggests that 1 undergoes rapid coil-to-helix and subsequent slow helix-to-helix transition in the presence of $(R)-3$. As mentioned with the ${ }^{1} \mathrm{H}$ NMR and IR spectra, 1 is able to undergo both Michael reaction and ester amidation in the presence of $(R)-3$. Because the former is kinetically much faster than the latter, the rapid coil-to-helix followed by slow helix-to-helix transitions can be well related with a rapid Michael reaction and a gentle ester amidation, respectively.

Polymer 1 showed the same tendency in the presence of chiral diamine, $(1 R, 2 R)$-(-)-diaminocyclohexane, whereas any changes in the CD spectra were a)
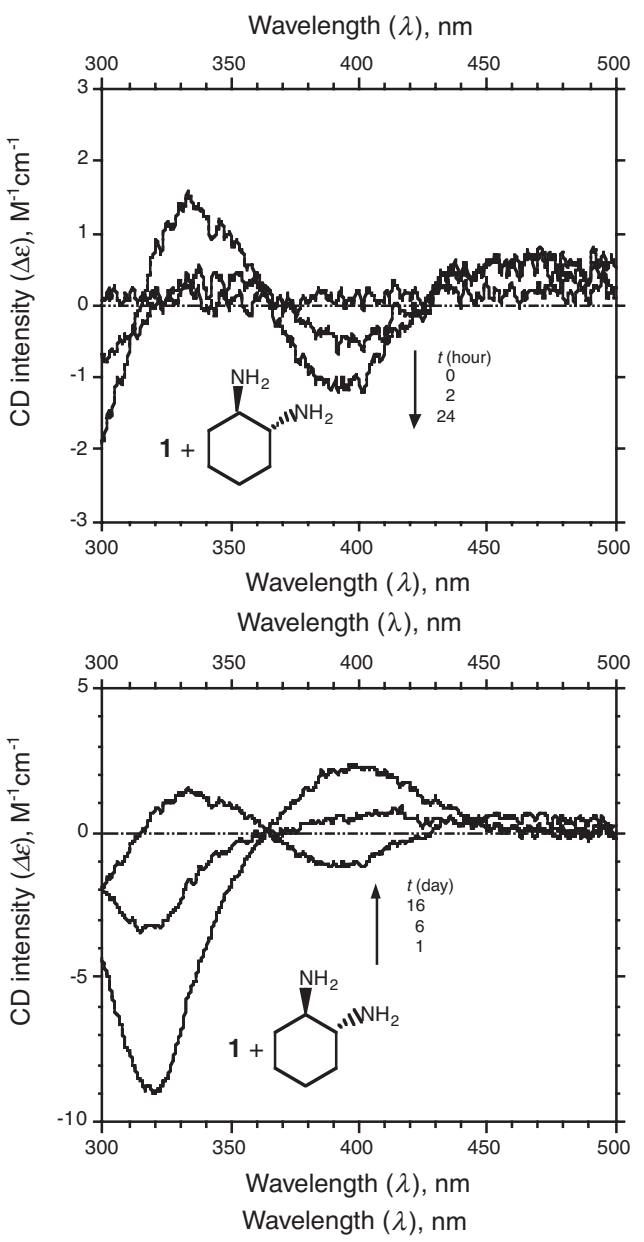

b)

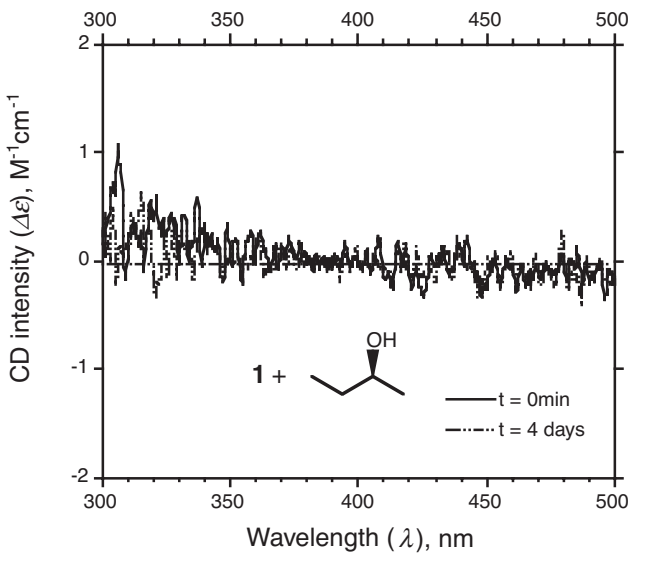

Figure 4. Changes in $C D$ spectra of 1 with aging time in the presence of a) excess $(1 R, 2 R)-(-)$-diaminocyclohexane and $\mathrm{b})$ excess $(S)-(+)$-2-butanol $\left([\mathrm{C}]_{0}=6.40 \times 10^{-4} \mathrm{M}\right.$, [Chiral molecule $] /[1]=300$, at $25^{\circ} \mathrm{C}$ in THF).

not detected in the presence of chiral alcohol, $(S)-(+)-$ 2-butanol (see Figure 4). Furthermore, when $(S)$-2amino-1-propanol, $(S)$-3, was added in place of the $(R)$-form, 1 exhibited a completely inverse CD signal, as expected (See Figure 5). As similar to the result of 1 added with $(R)-3$, the initial weak negative- $325 \mathrm{~nm}$ and positive- $380 \mathrm{~nm}$ Cotton bands were eventually inverted to the corresponding intense positive- $325 \mathrm{~nm}$ and negative- $380 \mathrm{~nm}$ Cotton bands, respectively, with 

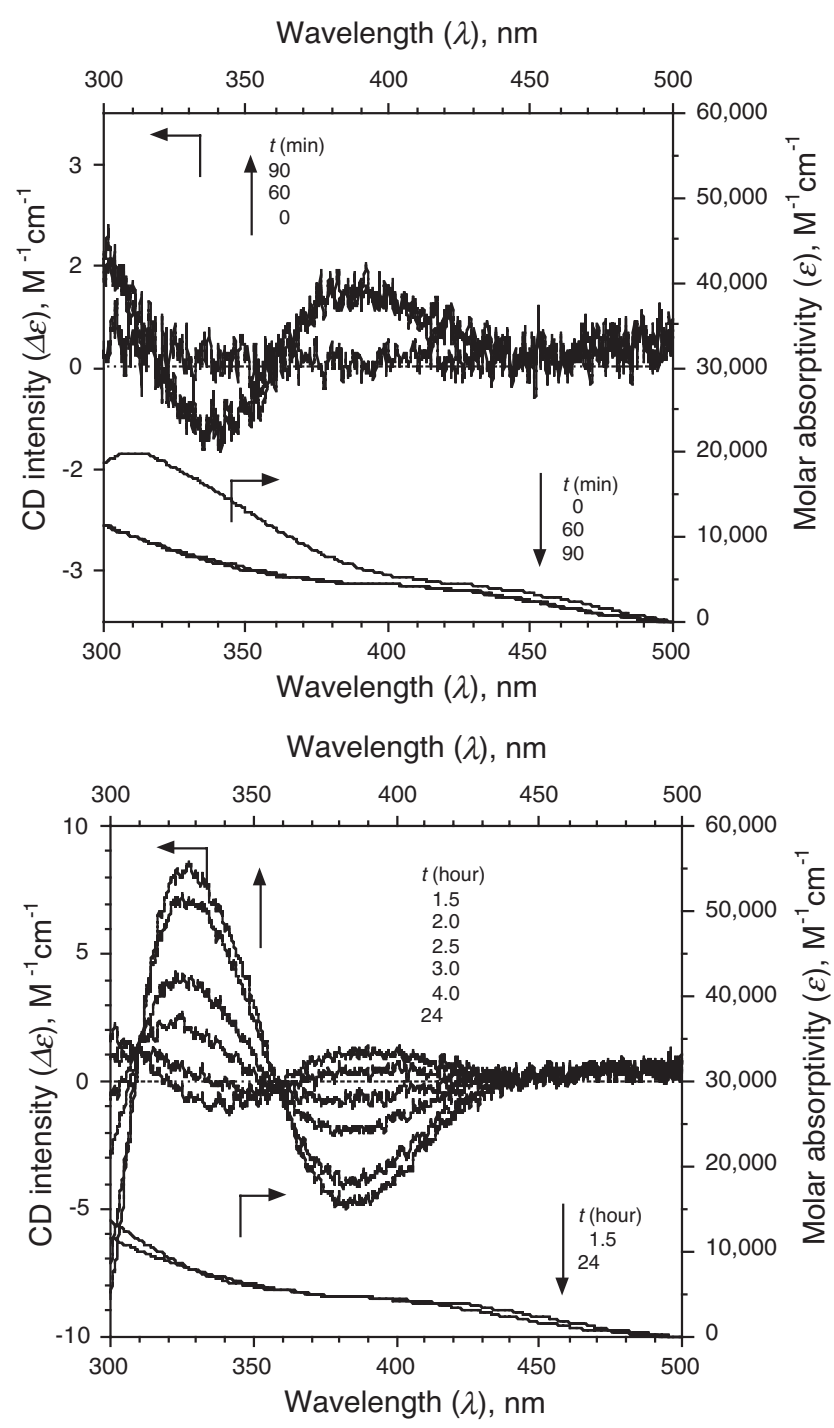

Figure 5. Changes in UV-vis and CD spectra of 1 with aging time in the presence of excess $(S)$-2-amino-1-propanol $\left([\mathrm{C}]_{0}=\right.$ $6.40 \times 10^{-4} \mathrm{M},[(S)$-2-amino-1-propanol $] /[1]=300$, at $25^{\circ} \mathrm{C}$ in THF).

a prolonged reaction time with $(S)-3$, as shown in Figure 6. Chiral nuclueophiles containing two $\mathrm{NH}_{2}$ or $\mathrm{OH}$ and $\mathrm{NH}_{2}$ groups may thus be needed to effectively induce a helical structure to 1 .

It should be noted that, as shown in Figure 1a, the broad ${ }^{1} \mathrm{H}$ NMR peaks in the range $2.35-3.00 \mathrm{ppm}$ due to amine $(j)$ and hydroxy $(n, w)$ protons significantly shifted to down field relative to those of the original $(R)-3$ which appeared in the range 2.15$2.60 \mathrm{ppm}$ in THF- $d_{8}$. This indicates the existence of the intramolecular hydrogen bonding between the side chains. Masuda et al. reported that the helical conformation of $\operatorname{poly}(\mathrm{N}$-propargylalkylamide) is stabilized by intramolecular hydrogen bonds between the side chains, and the polymer shows a relatively high viscosity index $\left(\alpha=0.91\right.$ in $\mathrm{THF}$ at $\left.40^{\circ} \mathrm{C}\right)$, indicating semi-flexible chain. ${ }^{9}$ Actually, certain poly $(N$-propargylamides) derivatives were able to switch their helix

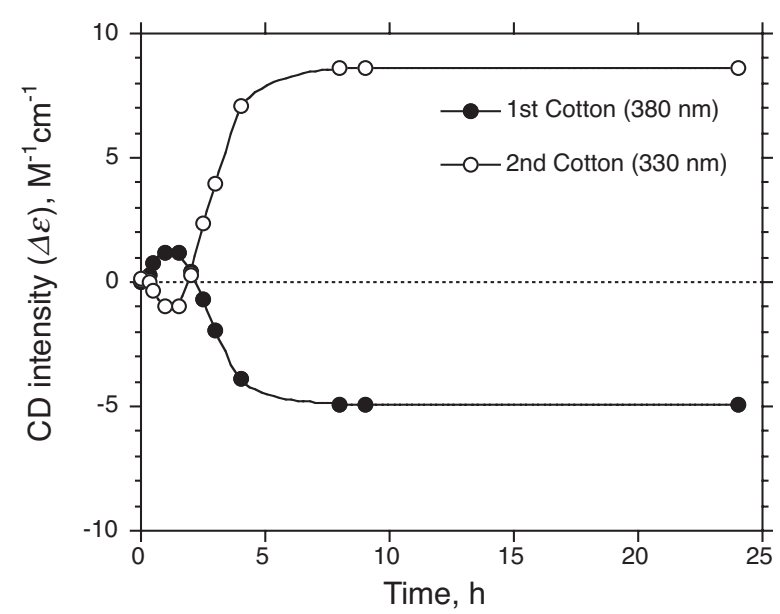

Figure 6. Time dependency of Cotton effects of 1 treated with (S)-2-amino-1-propanol.

sense in response to external stimuli such as solution temperature and polar protic solvents. ${ }^{15,17}$ The initial $\alpha$ value $\left(0.67\right.$ in $\mathrm{THF}$ at $30^{\circ} \mathrm{C}$, typical for random coiled conformation) of 1 significantly increased up to 0.93 , typical for semi-flexible conformation, after the treatment with $(R)-3$, indicating its greatly enhanced main-chain rigidity due to intramolecular hydrogen bonds. Indeed, in order to weaken and tune these intramolecular hydrogen bonds, when several $\mu \mathrm{L}$ of water was added to the THF solution $(3.0 \mathrm{~mL})$ of 1 treated with $(R)-3$, the $\mathrm{CD}$ signal was readily inverted and recovered to the initial helix sense, as shown in Figure 7. Polymer 1 is thus potentially applicable to the chiroptical switching induced by various chiral nucleophiles and sensory systems in response to a trace amount of water.

\section{CONCLUSION}

A new polyacetylene bearing benzylidene malonate group, poly[4-\{(diethyl- $\beta$-malonyl)vinyl $\}$ phenylacetylene], 1 , was designed as a prochiral reacting polyacetylene intermediate to provide helix induction and inversion abilities with chiral nucleophiles. Polymer 1 underwent both Michael reaction and amidation in the presence of $(R)-3$ to afford polymers with extremely complicated and optically active sites in the side chains. The rapid coil-to-helix followed by slow helix-to-helix transitions of 1 chain were well related with Michael reaction and ester amidation, respectively. Although the inverted helical conformation was fairly stabilized by intramolecular hydrogen bonds between the extremely complicated and optically active side chains, the helical sense was readily recovered to the initial screw sense state by adding a very little amount of water. Polymer 1 is thus applicable to a new type of chiroptical switching induced by various 
a)
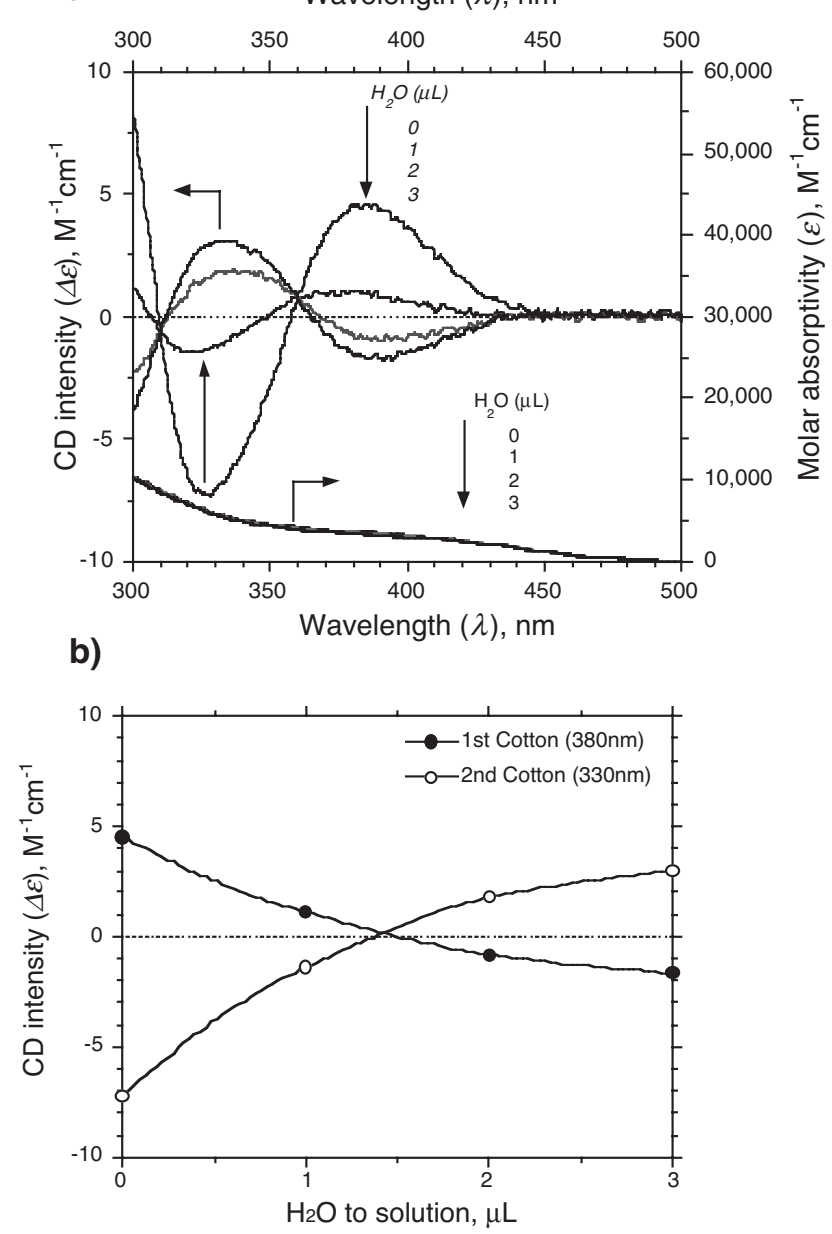

Figure 7. a) Changes in UV-vis and CD spectra of 1 treated with excess (R)-2-amino-1-propanol upon addition of water $\left([\mathrm{C}]_{0}=6.40 \times 10^{-4} \mathrm{M},[(R)\right.$-2-amino-1-propanol $] /[1]=300$, at $25^{\circ} \mathrm{C}$ in THF). b) Water amount dependency of Cotton effects of 1 treated with $(R)$-2-amino-1-propanol.

chiral nucleophiles and sensory systems to detect a trace amount of water. Polymer 1 may be a prototype of prochiral polymeric reacting intermediate to prove the idea of preparing various functional and biomimetic helical polymers in future.

Acknowledgment. We thank Profs. Kotohiro Nomura and Tamaki Nakano for fruitful discussion. This research was supported by grant-in-aid for scientific researches in a priority area "super-hierarchical structures" (No. 446) from the ministry of education, culture, sports, science and technology, Japan. MF is acknowledged in part for grants from the ministry of education, culture, sports, science and technology for grant-in-aid for scientific research, "design, synthesis, novel functionality of nanocircle and nanorod conjugating macromolecules (16205017)" and "experimental tests of macroscopic parity violation effects at polymer levels (16655046).

\section{REFERENCES}

1. C. F. Bernasconi and M. W. Stronach, J. Am. Chem. Soc., 113, 2222 (1991).

2. D. A. Evans, T. Rovis, M. C. Kozlowski, and J. S. Tedrow, J. Am. Chem. Soc., 121, 1994 (1999).

3. W. Zhuang, T. Hansen, and K. A. Jørgensen, Chem. Commun., 347 (2001).

4. G. Cardillo, L. Gentilucci, M. Gianotti, H. Kim, R. Perciaccante, and A. Tolomelli, Tetrahedron: Asymmetry, 12, 2395 (2001).

5. J. M. Betancort, K. Sakthivel, R. Thayumanavan, and C. F. Barbas III, Tetrahedron Lett., 42, 4441 (2001).

6. G. Cardillo, S. Fabbroni, L. Gentilucci, M. Gianotti, R. Perciaccante, and A. Tolomelli, Tetrahedron: Asymmetry, 13, 1407 (2002).

7. J. Zhou and Y. Tang, J. Am. Chem. Soc., 124, 9030 (2002).

8. J. J. L. M. Cornelissen, J. J. J. M. Donners, R. de Gelder, W. S. Graswinckel, G. A. Metselaar, A. E. Rowan, N. A. J. M. Sommerdijk, and R. J. M. Nolte, Science, 293, 676 (2001).

9. R. Nomura, J. Tabei, and T. Masuda, J. Am. Chem. Soc., 123, 8430 (2001).

10. H. Onouchi, K. Maeda, and E. Yashima, J. Am. Chem. Soc., 123, 7441 (2001).

11. B. S. Li, K. K. L. Cheuk, F. Salhi, J. W. Y. Lam, J. A. K. Cha, X. Xiao, C. Bai, and B. Z. Tang, Nano Lett., 1, 323 (2001).

12. R. Nonokawa and E. Yashima, J. Am. Chem. Soc., 125, 1278 (2002).

13. T. Aoki, T. Kaneko, N. Maruyama, A. Sumi, M. Takahashi, T. Sato, and M. Teraguchi, J. Am. Chem. Soc., 125, 6346 (2003).

14. K. K. L. Cheuk, J. W. Y. Lam, J. Chen, L. M. Lai, and B. Z. Tang, Macromolecules, 36, 5947 (2003).

15. J. Tabei, R. Nomura, and T. Masuda, Macromolecules, 36, 573 (2003).

16. K. K. L. Cheuk, J. W. Y. Lam, L. M. Lai, Y. Dong, and B. Z. Tang, Macromolecules, 36, 9752 (2003).

17. J. Tabei, R. Nomura, F. Sanda, and T. Masuda, Macromolecules, 37, 1175 (2004).

18. H. Nakashima, J. R. Koe, K. Torimitsu, and M. Fujiki, J. Am. Chem. Soc., 123, 4847 (2001).

19. H. Onouchi, T. Miyagawa, A. Furuko, K. Maeda, and E. Yashima, J. Am. Chem. Soc., 127, 2960 (2005). 